\title{
Article
}

\section{Correlation of the predisposition of Chinese children to cerebral palsy with nucleotide variation in pri- miR-124 that alters the non-canonical apoptosis pathway}

\author{
Hui $\mathrm{LI}^{1, \#}$, Xiu-li WANG ${ }^{2, \#}$, Yan-qiu WU ${ }^{1}$, Xiu-mei $\mathrm{LIU}^{1}$, Ai-min $\mathrm{LI}^{1,}$ * \\ ${ }^{1}$ Department of Pediatrics, The Affiliated Yantai Yuhuangding Hospital of Qingdao University, Yantai 264000, China; ${ }^{2}$ Department of \\ Gynecology, The Affiliated Yantai Yuhuangding Hospital of Qingdao University, Yantai 264000, China
}

\begin{abstract}
Cerebral palsy (CP) is a group of non-progressive motor impairment syndromes caused by brain lesions during development. Herein, we investigated the relationship between nucleotide variations in a miRNA coding region and the predisposition of Chinese children to cerebral palsy. A total of $233 \mathrm{CP}$ patients and 256 healthy participants were enrolled, and 60 children were selected from each group for plasma miRNA detection. We screened the coding regions of pri-miR-124-1, -2, and -3 using PCR and sequencing. The expression of miR-124 was determined by qRT-PCR. Luciferase assays and Western blots were used to confirm the regulation of target genes by miR-124. The function of miR-124 was further identified in SH-SY5Y cells by detecting cell viability and apoptosis. We revealed that the rare alleles $\mathrm{T}$ of $\mathrm{rs} 3802169$ and $\mathrm{G}$ of $\mathrm{rs} 191727850$ were found to be associated with an increased risk of cerebral palsy $(\mathrm{OR}=3.71$, $95 \% \mathrm{Cl}$ 1.74-7.92 and $\mathrm{OR}=2.18,95 \% \mathrm{Cl}$ 1.36-3.49, respectively). We further showed that the levels of mature miR-124 were downregulated by the C-to-T variation in vitro. More importantly, the reduction of miR-124 resulting from the C-to-T change led to the lessefficient inhibition of the target genes ITGB1, LAMC1 and BECN1, which may play important roles during the development of the nervous system. Meanwhile, the reduction in the expression of miR-124 was also related to the increased nuclear translocation of apoptosis-inducing factor (AIF) under oxidative stress, thereby inducing more cell apoptosis. Our results suggest that one functional polymorphism in pri-miR-124-1 might contribute to the genetic predisposition of Chinese children to cerebral palsy by disrupting the production of miR-124, which consequently interfered in the expression and function of the target genes of miR-124.
\end{abstract}

Keywords: cerebral palsy; miR-124; polymorphism; non-canonical apoptosis pathway; Chinese children

Acta Pharmacologica Sinica (2018) 39: 1453-1462; doi: 10.1038/aps.2017.211; published online 17 May 2018

\section{Introduction}

Cerebral palsy $(\mathrm{CP})$ is a group of non-progressive motor impairment syndromes caused by lesions of the brain that arise early in development ${ }^{[1]}$. Risk factors that lead to this condition such as preterm birth, intrauterine infection, intrauterine growth restriction, and perinatal stroke have been identified $^{[2,3]}$. Furthermore, genetic makeup is also related to the predisposition to cerebral damage by influencing the response to the presence of these risk factors ${ }^{[4,5]}$. Several genetic variants that cause Mendelian forms of CP have been described by other researchers ${ }^{[6]}$, and variants in the IL- 6 coding region

\footnotetext{
\# These authors contributed equally to this work.

*To whom correspondence should be addressed.

E-mail woxinfeiyang_7777@126.com

Received 2017-08-22 Accepted 2017-12-14
}

have also been reported to contribute to enhanced genetic predisposition to $\mathrm{CP}^{[7]}$.

MicroRNAs are a group of negative regulators of gene expression that repress gene expression by targeting the 3'UTR of mRNA. A group of miRNAs expressed in the central nervous system are dynamically regulated both during physiological brain development and in vitro neural differentiation of stem cells, indicating that they have a significant contribution to neural development and function ${ }^{[8,9]}$. Meanwhile, abnormal miRNA levels have been found to be related to human nervous system diseases such as NTD, schizophrenia, and Alzheimer's disease ${ }^{[10-12]}$. miR-124 is a brain-enriched miRNA that is derived from three independent genes (miR-124-1, -2, and -3 ) located on chromosome 8 or 20 . Interestingly, knockout of just the miR-124- 1 gene in mice resulted in a significant reduction of the mature miR-124 level, defective neuronal sur- 
vival, and axonal outgrowth, as well as smaller brain size $\mathrm{e}^{[13]}$. Jenn-Yah et al have reported that the miR-124 gene is one of the most abundantly expressed in the adult nervous system and that it regulates neurite outgrowth during neuronal differentiation $^{[14]}$. However, it remains unknown whether the SNPs of miR-124 affect miRNA biogenesis and human genetic disease. BECN1 mRNA and protein are abundantly expressed in neurons and glia in human brains ${ }^{[15,16]}$. Beclin- 1 is a protein encoded by the BECN1 gene. It regulates the autophagyinduced activity of $\mathrm{Vps} 34^{[17]}$ and plays an important role in the formation of autophagosomes ${ }^{[18]}$. The integrin $\beta 1$ gene (ITGB1) encodes $\beta 1$ integrin, which could also promote neurite outgrowth ${ }^{[19]}$. The laminin $\mathrm{c} 1$ gene (LAMC1) could encode ECM-associated proteins. In addition, mutations in ECMassociated proteins lead to cortical neuron lamination defects, including fragility of the basement membrane and detachment of radial glia from the basement membrane ${ }^{[20]}$. In this study, BECN1, ITGB1 and LAMC1 genes were selected as the target genes to predict the functional consequence of enhanced processing of miR-124.

Increasing evidence has shown that single nucleotide polymorphisms (SNPs) or mutations in miRNA coding regions may alter miRNA expression and/or maturation and be related to the occurrence of diseases ${ }^{[21,22]}$. Meanwhile, some of the alterations in expression are detectable in serum ${ }^{[23]}$. In this study, to explore the relationship between SNPs in the miRNA coding region and the occurrence of $\mathrm{CP}$, we detected the serum levels of 11 candidate miRNAs in $56 \mathrm{CP}$ patients. The coding regions of 3 altered miRNAs were sequenced, and one SNP was found to be related to the genetic predisposition to $\mathrm{CP}$ by reducing the level of mature miRNA.

\section{Materials and methods Subjects}

In this study, $233 \mathrm{CP}$ patients (103 girls and 130 boys with mean ages $\pm S D$ of $18.9 \pm 13.8$ months) were chosen from the Rehabilitation Center of the Affiliated Yantai Yuhuangding Hospital of Qingdao University from 30th March 2012 to 28th September 2015. In addition, 256 healthy control participants (112 girls and 144 boys with mean ages \pm SD of $19.2 \pm 11.7$ months) were chosen from Department of Pediatrics at the same hospital. Of these, $60 \mathrm{CP}$ patients and 60 control patients were selected for plasma miRNA detection. The age of the selected children ranged from 5 months to 36 months. All subjects were Han Chinese from North China, and written informed consent to participate in this study was provided by the children's parents. Child neurologists diagnosed and classified the CP by clinical examination or by using medical records, including brain imaging, according to the guidelines proposed by the Surveillance of CP in Europe network ${ }^{[24]}$. Children suffering from hypotonia, ataxia, myopathy, a genetic syndrome or a chromosomal anomaly were excluded. Blood samples were collected in tubes containing EDTA by skilled nurses on the second day of hospitalization. Plasma was separated from the $500 \mu \mathrm{L}$ blood sample by centrifugation (1500 $\times g$ for $15 \mathrm{~min}$ ) at $4^{\circ} \mathrm{C}$.

\section{DNA extraction and genotyping}

Genomic DNA was extracted from peripheral blood samples from the CP patients and the controls by using the QIAamp DNA Blood Mini kit (Qiagen, Hilden, Germany) following the manufacturer's instructions. DNA quantity and quality were determined using NanoDrop 2000 ultraviolet-visible spectrophotometry (Thermo Fisher Scientific, Wilmington, DE, USA). The PCR products were sequenced in the forward direction with the ABI 3730xl sequencing platform. The sequencing results were analyzed by using DNAMAN and BLAST. The primers were as follows: miR-124-1 forward 5'-TCТTCTAСССАССССТСТTCC-3', reverse 5'-AATCTGCACACACAAGCACTC-3' and miR-124-2 forward 5'-GGGTAATTAATTTGGATTTACGTCGTTAT-3', reverse 5'-CGTAAAAATATAAACGATACGTATACCTACGT-3'. The genomic DNA fragment amplified by the primers contains a single nucleotide polymorphism (SNP). The SNP genotypes of the genomic DNA samples were determined by sequence analysis.

\section{RNA isolation}

Total plasma RNA was extracted using the TRIzol LS reagent (Invitrogen, Carlsbad, CA, USA) following the manufacturer's instructions. Briefly, $250 \mu \mathrm{L}$ of plasma was added to $750 \mu \mathrm{L}$ of TRIzol reagent and incubated at room temperature for 5 min. Chloroform was added to the samples, and they were vigorously mixed and incubated at room temperature for 5 min. Following incubation, the samples were centrifuged at $12000 \times g$ for $15 \mathrm{~min}$ at $4^{\circ} \mathrm{C}$. RNA was precipitated from the aqueous phase by addition of isopropyl alcohol to a fresh tube containing the supernatant in the aqueous phase. The integrity of the RNA was tested by spectroscopic analysis.

\section{RNA secondary structure prediction}

The secondary structure of the 316-bp pri-miR-124 including the mutation site was predicted using the RNAfold web server (http:/ / rna.tbi.univie.ac.at/cgi-bin/RNAWebSuite/RNAfold. cgi).

\section{miR-124 expression vectors}

To construct the miR-124 expression vectors, we engineered expression plasmids with 425 nt of pre-miR-124-1 and its flanking regions, which included two SNP sites. The primiR-124-1 DNA sequences were amplified through PCR from the cDNA of different genotypes. The following primers of pri-miR-124-1 were used: forward 5'-GGGGTACCGAAGCAGAGGGCGACATGG-3' and reverse 5'-AAAACTGCAGGCAGTCTGCCAGTCTCTGGATGC-3'. The following primers of pri-miR-124-2 were used: forward 5'-TCTTCTACCCACCCCTCTTCC-3' and reverse 5'-AATCTGCACACACAAGCACTC-3'. The PCR products were cloned into the pcDNA3.1 vector (Invitrogen, Carlsbad, CA, USA). The sequences of these vectors were confirmed by direct sequencing with the ABI 3730xl sequencing platform.

\section{Real-time reverse transcriptase quantitative PCR}

The expression levels of miR-124-3p and $-5 p$ and the plasma 
levels of the miR-124 genes were determined by qRTPCR using TaqMan Universal PCR Master Mix (Applied Biosystems, Foster City, CA, USA) together with miRNAspecific TaqMan MGB probes. U6 snRNA was used for normalization. Each sample in each group was measured in triplicate, and the experiment was repeated at least three times.

\section{Dual luciferase assay}

The full length of the 1031-bp BECN1 3'UTR, the full length of the 2879-bp LAMC1 3'UTR and a 668-bp segment of ITGB1 were cloned separately into the pmirGLO vector downstream of the firefly luciferase coding region (Promega, Madison, WI, USA) to generate luciferase reporter vectors. For the luciferase reporter assays, HEK293T cells were seeded in 48-well plates. miR-124 expression vectors and luciferase reporter vectors were co-transfected by using Lipofectamine 2000 (Invitrogen, Carlsbad, CA, USA) and different genotypes of pri-miR-124 (CC, CT or TT). The cells were harvested 48 $\mathrm{h}$ after transfection, and the luciferase activity was assayed using the Dual-Luciferase Assay kit (Promega, Madison, WI, USA). Each treatment was performed in triplicate in three independent experiments. The results were expressed as relative luciferase activity (Firefly LUC/Renilla LUC).

\section{Western blot}

The proteins were extracted with an appropriate amount of protein lysis buffer (Beyotime, Shanghai, China) in each plate. After centrifugation at $15000 \times g$ and $4{ }^{\circ} \mathrm{C}$, the supernatant was collected. A $25 \%$ volume of $5 \times$ loading buffer was added to the protein samples before the protein was loaded, and the samples were denatured at $100^{\circ} \mathrm{C}$ for $5 \mathrm{~min}$. Equal amounts of protein $(20 \mu \mathrm{g})$ were dissolved using $12 \%$ SDS-PAGE and then transferred onto PVDF membranes, followed by the addition of blocking buffer (TBST containing $5 \%$ bovine serum albu$\mathrm{min}$ ) at room temperature for $1 \mathrm{~h}$. After that, the PVDF membranes were incubated with the appropriate primary antibodies overnight at $4^{\circ} \mathrm{C}$, and the membranes were washed 3 times with Tris-buffered saline with Tween-20 (TBST) followed by an incubation for $1 \mathrm{~h}$ with HRP-conjugated secondary antibody at room temperature. The PVDF membranes were developed by an enhanced chemiluminescent (ECL) system (Li-cor, Lincoln, NE, USA) and exposed to UVP chemiluminescence (Biolite LLC, Upland, CA, USA). Protein quantification was performed by AlphaView SA software by calculating the band densities.

\section{Apoptosis analysis}

Cell apoptosis was detected using annexin V-FITC and propidium iodide (PI) staining and analyzed by flow cytometry. Briefly, the SH-SY5Y cells $\left(\sim 1 \times 10^{5}\right)$ were transfected with primiR-124-CC, -CT or -TT expression vectors for $48 \mathrm{~h}$, while SH-SY5Y cells in control group was only treated with DMEM medium. Then, the cells were harvested, washed with PBS solution, cultured with annexin V-FITC and PI at room temperature for $15 \mathrm{~min}$ in the dark, and analyzed using a FACS
Calibur (BD Bioscience) with a minimum of 10000 cells in each sample.

\section{Cell proliferation assay}

Cell viability was determined through MTT assay. Briefly, human neuroblastoma SH-SY5Y cells were seeded in 96-well plates at a low density of $5 \times 10^{3}$ cells per well in DMEM culture and allowed to attach overnight. The cells were then transfected with different genotype mir-124 expression vectors for $48 \mathrm{~h}$ at $37^{\circ} \mathrm{C}$ in $5 \% \mathrm{CO}$. Then, $20 \mu \mathrm{L}$ of MTT $(5 \mathrm{mg} / \mathrm{mL})$ (Sigma, St Louis, MO, USA) was added into each well at 4 different time points $(0 \mathrm{~h}, 24 \mathrm{~h}, 48 \mathrm{~h}, 72 \mathrm{~h})$ after transfection, and the cells were incubated for another $4 \mathrm{~h}$. The optical density was recorded at $570 \mathrm{~nm}$ after the DMSO addition using a microplate reader (Spectra Max M2, Molecular Devices, USA).

\section{Oxidative stress assay}

To mimic oxidative stress conditions, SH-SY5Y cells were transfected with miR-124 inhibitor for $24 \mathrm{~h}$ and then treated with $0.5 \mathrm{mmol} / \mathrm{L} \mathrm{H}_{2} \mathrm{O}_{2}$ for $1 \mathrm{~h}$ at $37^{\circ} \mathrm{C}$. The cells were centrifuged and washed to remove the hydrogen peroxide. Then, $10 \mu \mathrm{L}$ of annexin V-FITC and $5 \mu \mathrm{L}$ of PI were added, and the cells were cultured in the dark at $4{ }^{\circ} \mathrm{C}$ before being analyzed using a FACS Calibur (BD Bioscience, USA).

\section{ELISA}

The plasma expression levels of the BECN1 protein were determined by enzyme-linked immunosorbent assay (ELISA) kits (eBioscience Inc, San Diego, CA, USA) following the manufacturers' instructions.

\section{Statistical analysis}

For gene analysis, a test for Hardy-Weinberg equilibrium was performed on the allele and genotype frequency analysis using the SHEsis online software platform (http://analysis.bio-x. $\mathrm{cn})$. The relative risk was approximated by the estimate of the odds ratio (OR). All ORs were adjusted for age and sex using logistic regression models. The tested correlation between BECN1 levels and miR-124 expression was analyzed using $X^{2}$-analysis. Independent analysis of the differences between groups was performed with $t$-tests. The data are reported as the mean $\pm S D$. Comparisons between multiple groups were performed by one-way ANOVA followed by the StudentNewman-Keuls (SNK) method. All statistical analyses were analyzed with the SPSS Statistical Package version 16. $P<0.05$ was considered statistically significant.

\section{Results}

\section{The genotypic distribution of SNPs in CP patients and controls} miR-124 is a brain-enriched miRNA, the aberrant expression of which contributes to pathological conditions involving the central nervous system. To determine whether there are relationships between $\mathrm{CP}$ genetic predisposition and nucleotide variations in the miR-124 coding region, we first screened the sequences of pri-miR-124-1, -2 and -3 in $233 \mathrm{CP}$ patients and 256 healthy controls. Differences in allele frequencies 
Table 1. Genotype frequency and allele frequencies of SNP rs531564, rs73662598, rs3802169, rs569595042, rs191727850 and rs568537328 in miR-124-1 and - 2 in CP patients and control participants.

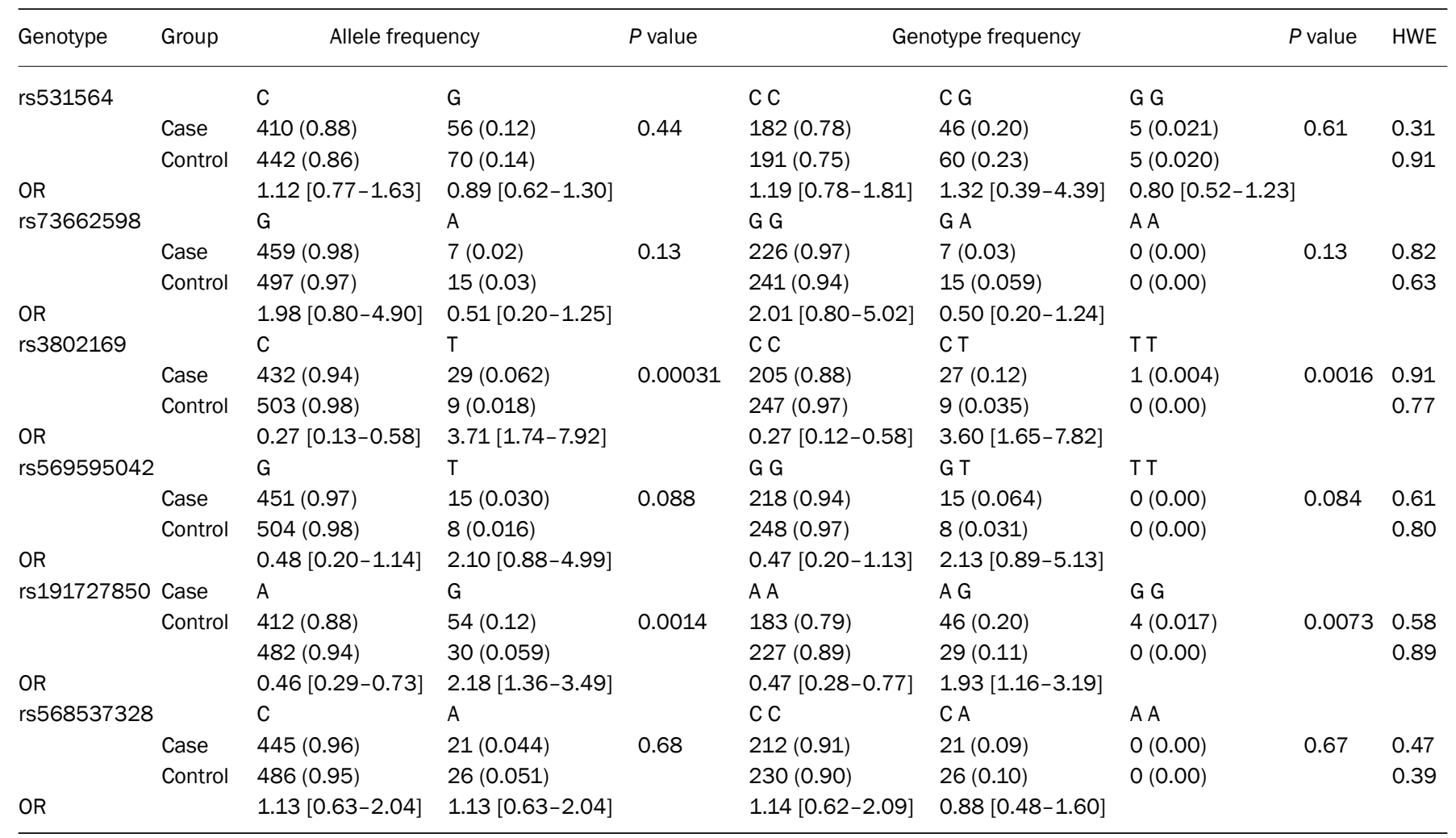

and genotype frequencies between $\mathrm{CP}$ patients and healthy controls were calculated (Table 1). As shown in Table 1, the distribution of allele frequencies and genotype frequencies of SNPs rs531564, rs73662598 and rs569595042 in the controls and patients was in Hardy-Weinberg equilibrium $(P>0.05)$. Significant differences were found in the allele and genotype distribution of rs3802169 and rs191727850 within pri-miR-124-1 and pri-miR-124-2 in CP patients compared with controls $(P<0.05)$. Meanwhile, we concluded that $\mathrm{C} / \mathrm{T}$ heterozygosity of rs3802169 $(\mathrm{OR}=3.60,95 \% \mathrm{CI}=1.65-7.82)$ and A/G heterozygosity of rs191727850 (OR=1.93, 95\% CI=1.16-3.19) were associated with an increased risk of $\mathrm{CP}$.

\section{Prediction of the effects of SNPs rs3802169 and rs191727850} on the structure of pri-miR-124-1 and -2

To understand the functions of these two SNPs, we first analyzed the predicted secondary structure and molecular stability of pri-miR-124-1 and -2. As shown in Figure 1A, the rare allele $\mathrm{T}$ of $\mathrm{rs3802169}$ at the position -78 relative to pre-miR-124-1 causes an apparent change in the secondary structure and a lower predicted $\Delta \mathrm{G}$ from $-113.80 \mathrm{kcal} / \mathrm{mol}$ to $-114.60 \mathrm{kcal} / \mathrm{mol}$. Furthermore, the expression of mature miR-124-3p and -5p was up-regulated in the HEK293T cells transfected with the pri-miR-124-1-TT expression vector (Figure 1B). The rare allele $\mathrm{G}$ of rs191727850 at the position of -62 relative to pre-miR-124-2 did not change the predicted secondary structure of pri-miR-124-2 nor the predicted $\Delta \mathrm{G}$
(Figure 2A). In addition, the relative miR-124-3p and $-5 p$ expression levels in the AA, AG and GG genotype groups are shown in Figure 2B. We concluded that there were no significant differences in the expression levels of mature miR-124-3p and $-5 p$ after transfection with AA, AG and GG genotype pri-miR-124-1 expression vectors (Figure $2 \mathrm{~B}$ ).

\section{Prediction of miR-124-3p and relative luciferase activity in different groups}

Since the biological function of a miRNA mostly depends on what downstream genes it represses, we predicted the direct targets of miR-124-3p using the online bioinformatics tool TargetScan (http://www.targetscan.org). As shown in Figure 3A, miR-124-3p may repress BECN1 expression by targeting a 7-mer site in the 3'UTR of BECN1 mRNA. Subsequently, we constructed a dual-luciferase reporter vector using the full length of the BECN1 3'UTR as the 3'UTR of firefly luciferase. The reporter vector was co-transfected with the miR-124-3p mimic or inhibitor into HEK293T cells. The cells were lysed $48 \mathrm{~h}$ after transfection, and the luciferase activity was detected. As shown in Figure 3B, the relative luciferase activity was reduced by $45.2 \%$ by the miR-124-3p mimic and increased by $13.4 \%$ in the cells transfected with the miR-124-3p inhibitor. These results indicated that miR-124-3p repressed the luciferase activity by targeting the 3'UTR of BECN1. To examine whether endogenous BECN1 expression was repressed by miR-124-3p, HEK293T cells were transfected with 
A

rs3802169

Pri-miR-124-1 CC
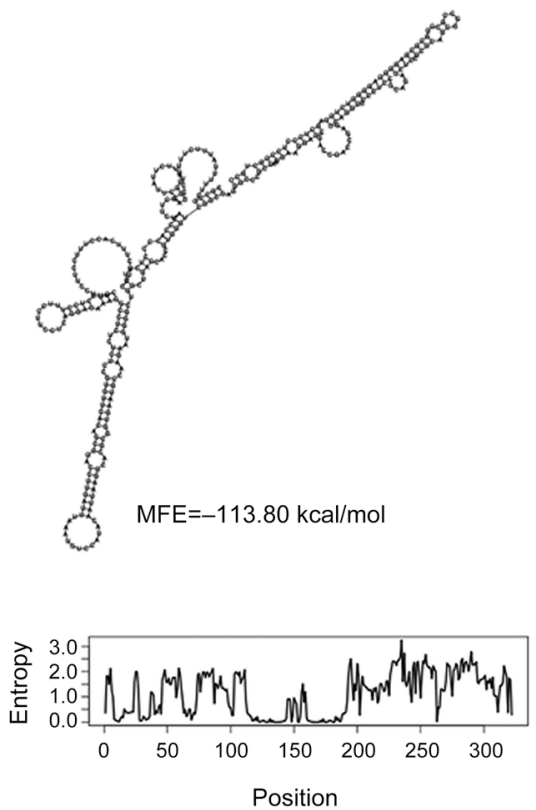

B

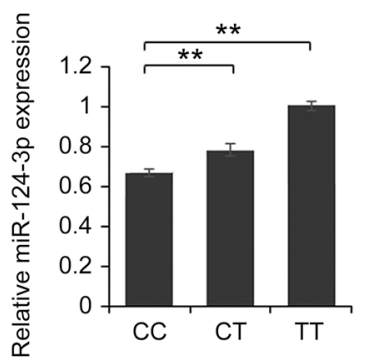

rs3802169

Pri-miR-124-1 TT
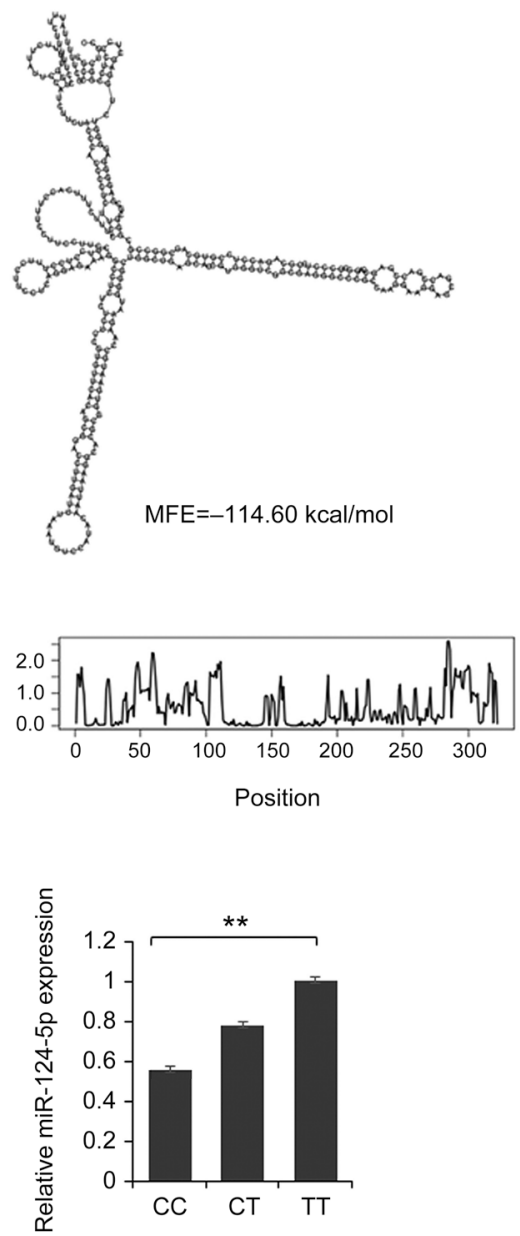

Figure 1. The $\mathrm{C}$ to $\mathrm{T}$ change in rs3802169 enhances the stability of pri-miR-124-1 and up-regulates miR-124 expression. (A) The predicted secondary structure of different genotypes of the 316-bp pri-miR-124-1. (B) Relative expression of mature miR-124-3p and -5p according to different genotypes of pri-miR-124-1. HEK293T cells were transfected with pri-miR-124-1-CC, pri-miR-124-1-CC and -TT or pri-miR-124-1-TT. RNA was extracted 48 h after transfection, and the level of mature miR-124 was determined by qRT-PCR.

miR-124-3p mimic or inhibitor. The cells were lysed $48 \mathrm{~h}$ after transfection, and the BECN1 expression level was determined by immunoblotting. As shown in Figure 3C, the BECN1 protein level was markedly reduced in cells transfected with the miR-124-3p mimic and up-regulated in cells transfected with the miR-124-3p inhibitor, which was consistent with the result of the dual-luciferase analysis.

To initiate a study of the functional consequence of enhanced processing of miR-124 for its target gene, we chose three miR-124-3p target genes. Among them, LAMC1 and ITGB1 were identified as endogenous targets of miR-124, both of which are highly expressed by neural progenitors and are related to neuronal differentiation. The full length LAMC1 3'UTR and a 668-bp segment of the ITGB1 3'UTR were separately cloned into the pmirGLO vector downstream of the firefly luciferase coding region to generate luciferase reporter vectors. These constructed reporters were transiently transfected into HEK293T cells together with an expression plas- mid containing one genotype of miR-124-1. Compared with cells transfected with pri-miR-124-1-CC, the activity of firefly luciferase was increased by $12.5 \%(P<0.01)$ or $47.0 \%(P<0.01)$ in cells co-transfected with pmirGLO-LAMC1 and pri-miR124-1-CT or -TT, respectively. The activity of firefly luciferase was decreased by $40.6 \%(P<0.01)$ in cells co-transfected with pmirGLO-ITGB1 and pri-miR-124-1-TT compared to the activity in cells transfected with pri-miR-124-1-TT. Meanwhile, the activity of firefly luciferase was significantly decreased by $20.5 \%$ and $39.8 \%$ in cells co-transfected with pmirGLO-BECN and pri-miR-124-CT or pri-miR-124-TT, respectively $(P<0.05$ or $P<0.01)$. These results indicate that the $\mathrm{C}$-to- $\mathrm{T}$ change in rs3802169 can inhibit target gene expression by up-regulating miR-124-1.

\section{Expression levels of LC3II and LC3I were determined by Western blot}

BECN1 plays an important role during autophagic pro- 
A

\section{rs191727850}

Pri-miR-124-2 AA
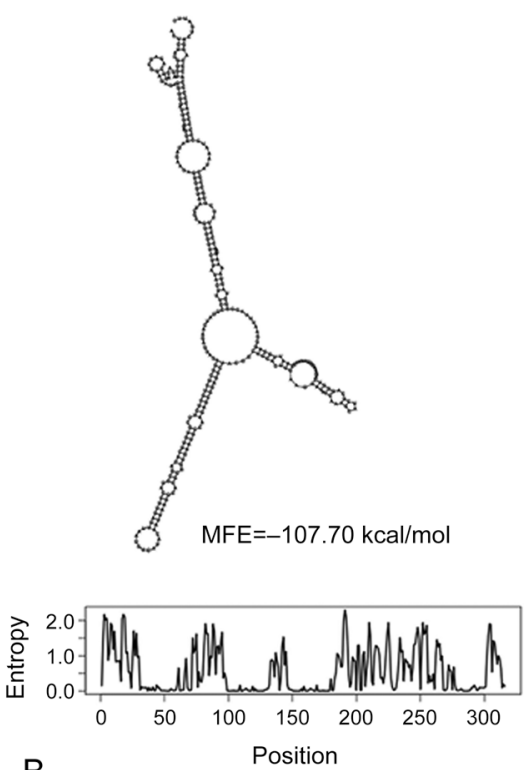

B

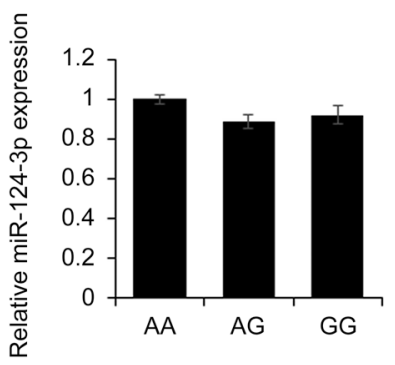

rs191727850

Pri-miR-124-2 GG
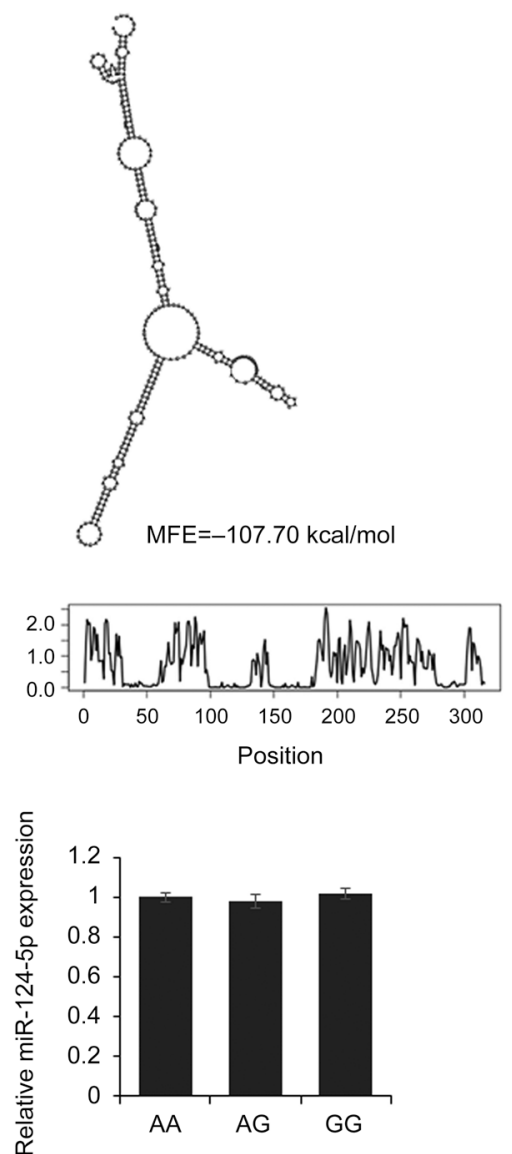

Figure 2. The A to G variation of rs 191727850 did not alter the predicted secondary structure of pri-miR-124-2 or the expression of miR-124. (A) The predicted secondary structure of different genotypes of the 322-bp pri-miR-124-2. (B) Relative expression of mature miR-124-3p and -5p according to different genotypes or pri-miR-124-2. HEK293T cells were transfected with pri-miR-124-2-AA, pri-miR-124-2-AA and -GG or pri-miR-124-2-GG. RNA was extracted $48 \mathrm{~h}$ after transfection, and the level of mature miR-124 was determined by qRT-PCR.

grammed cell death, and the LC3II/I value was used to evaluate the degree of autophagy. We detected the LC3II and LC3I levels in SH-SY5Y cells transfected with expression vector with different genotypes of pri-miR-124 through Western blot. As shown in Figure 4A, the level of the BECN1 protein continually increased in the cells transfected with pri-miR-124-CC, -CT and -TT. Meanwhile, the level of the LC3I protein was down-regulated in cells transfection with pri-miR-124-CT and -TT compared to the level in those transfected with primiR-124-CC. However, the level of the LC3II protein was upregulated in cells transfected with pri-miR-124, especially primiR-124-TT, suggesting an enhanced autophagy pathway.

Cell proliferation and apoptosis analysis of SH-SY5Y cells transfected with miR-124-CC, -CT and -TT

Subsequently, the cell viability and apoptosis of SH-SY5Y cells transfected with expression vectors with different genotypes of pri-miR-124 were examined through MTT assay and flow cytometry. As shown in Figure 4B, the relative viability of
SH-SY5Y cells transfected with the pri-miR-124-CC vector was $\sim 35 \%, \sim 73 \%$ and $\sim 85 \%$ at $24 \mathrm{~h}, 48 \mathrm{~h}$ and $72 \mathrm{~h}$, which are higher than the viability of cells treated with the pri-miR-124-CT vector $(\sim 33 \%, \sim 58 \%$ and $\sim 64 \%, P<0.05)$. In addition, we found a lower cell viability at $72 \mathrm{~h}(\sim 52 \%, P<0.05)$ in cells treated with the pri-miR-124-TT vector compared to cells transfected with the pri-miR-124-CC or -CT expression vectors. Flow cytometry revealed that SH-SY5Y cells transfected with primiR-124-CT and -TT expression vectors had higher apoptosis rates $(\sim 14.1 \%$ and $\sim 17.3 \%, P<0.05$ or $P<0.01$, respectively) than cells transfected with the pri-miR-124-CC expression vector $(\sim 9.8 \%)$ (Figure $4 \mathrm{C})$.

\section{Plasma miR-124 and BECN1 levels detected by qRT-PCR}

To further explore the function of the C-to-T variation in rs3802169 in vivo, the plasma miR-124 and BECN1 levels of $\mathrm{CP}$ patients and controls were detected through qRT-PCR and ELISA, respectively. As shown in Figure 5A, the plasma miR124 level of $\mathrm{CP}$ patients was higher than that of the control 
A

Position 118-125 of BECN1 3'UTR

Has-miR-124-3p

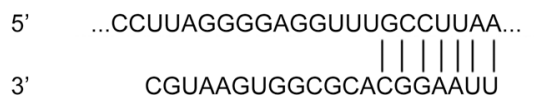

C

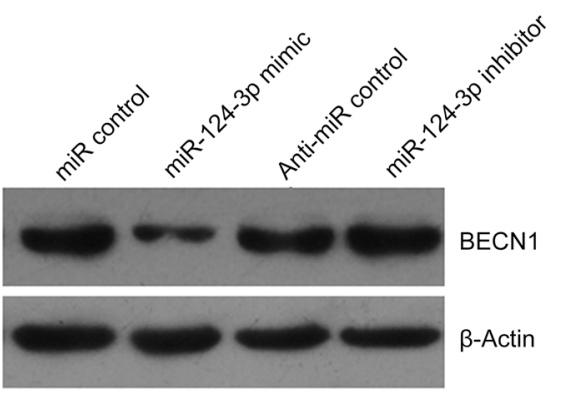

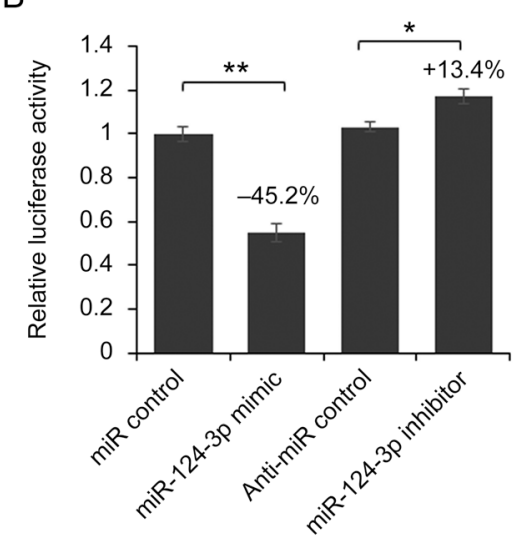

D
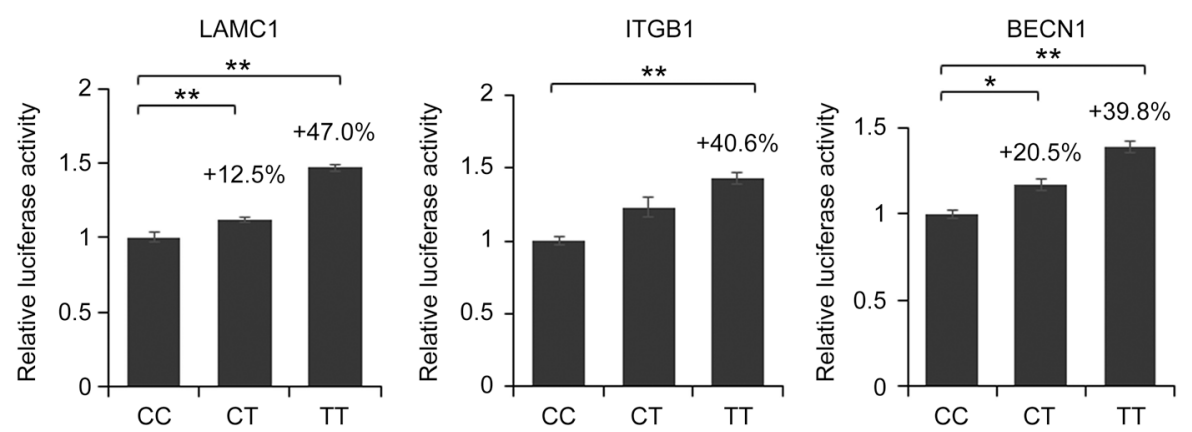

Figure 3. BECN1 is a direct target of miR-124. (A) The predicted direct interaction between miR-124-3p and the BECN1 3'UTR. (B) Dual-luciferase assay. HEK293T cells were transfected with the BECN1 3'UTR reporter vector and miR-124-3p mimic or inhibitor. The cells were lysed $48 \mathrm{~h}$ after transfection, and luciferase activity was detected. The results are represented as firefly luciferase activity/renilla luciferase activity. (C) Western blot analysis of BECN1 expression in HEK293T cells transfected with miR-124 mimic or inhibitor. (D) Dual luciferase assay. HEK293T cells were transfected with expression vectors containing different genotypes of pri-miR-124-1 and a reporter vector containing the 3'UTR of LAMC1, ITGB1 or BECN1. The cells were lysed $48 \mathrm{~h}$ after transfection, and luciferase activity was detected. ${ }^{*} P<0.05,{ }^{* *} P<0.01$, vs miR-124-CC vector group.

group $(P<0.05)$. However, the plasma level of the BECN1 protein was lower than that of the control group $(P<0.05$, Figure 5B). In addition, the result of the correlation analysis indicated a significant negative correlation between serum miR-124 and BECN1 levels in CP patients (Figure 5C).

Expression of AIF and the cell apoptosis analysis in control and with miR-124 inhibitor

Increased oxidants and decreased antioxidants are usually found in children with $\mathrm{CP}$ and contribute to the pathogenesis of cerebral injury ${ }^{[25]}$. Under oxidative stress, apoptosisinducing factor (AIF) will translocate from the mitochondria to the nucleus and induce cell apoptosis. To mimic the oxidative conditions, SH-SY5Y cells were transfected with miR-124 inhibitor for $24 \mathrm{~h}$ and then treated with $0.5 \mathrm{mmol} / \mathrm{L} \mathrm{H}_{2} \mathrm{O}_{2}$ for 1 h. As shown in Figure 6A, the AIF level was significant higher in the nucleus when the cells were treated with the miR-124 inhibitor. Meanwhile, the AIF level was reduced in the cytoplasm, and the total AIF expression was not changed. These results indicated that miR-124 inhibitor can up-regulate AIF cytoplasm-nucleus translocation under oxidant stress. The cell apoptosis assay indicated that the apoptotic cell number was markedly up-regulated when the cells were treated with the miR-124 inhibitor compared with the apoptotic cell number of the control (Figure 6B).

\section{Discussion}

Cerebral palsy is a group of non-progressive motor impair- 
A

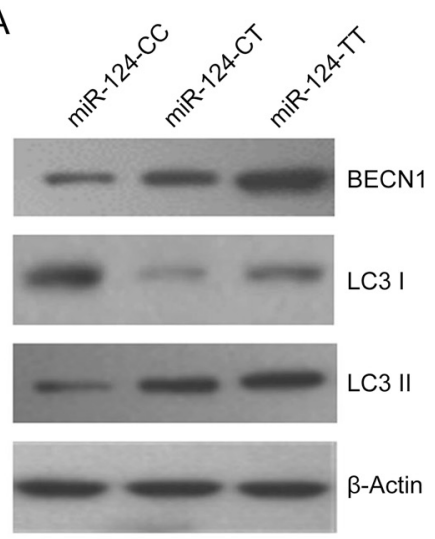

C

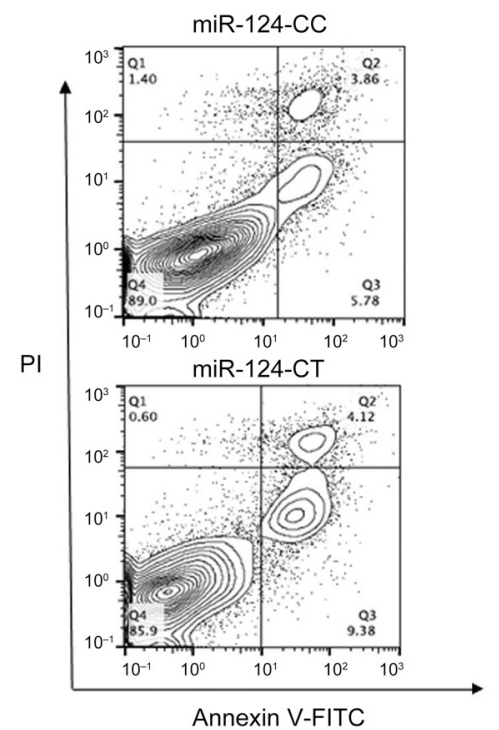

B
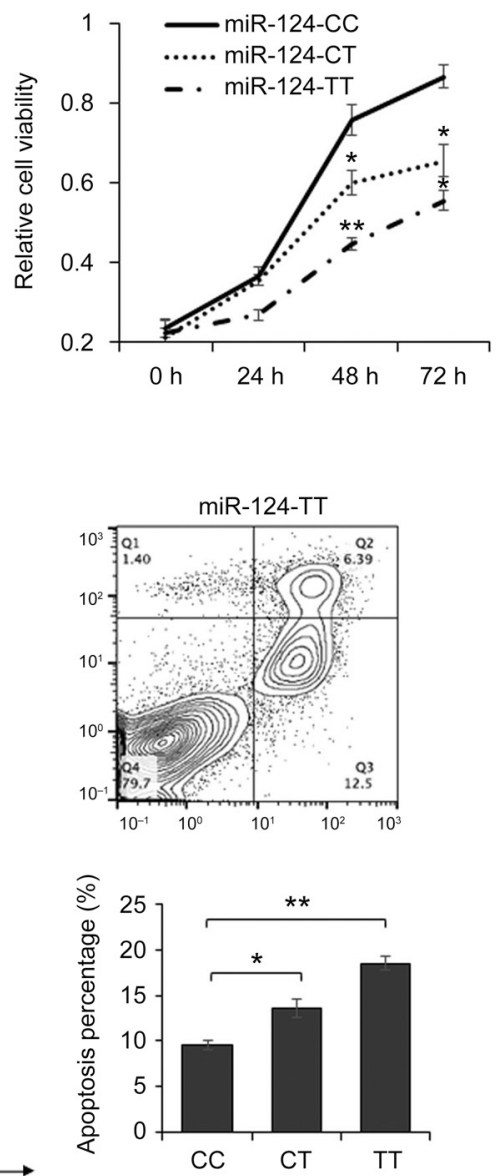

Figure 4. The $\mathrm{C}$ to $\mathrm{T}$ change in rs3802169 is related to reduced cell viability and enhanced apoptosis. (A) SH-SY5Y cells and SKNSH cells transfected with expression vectors containing different genotypes of pri-miR-124. Cells were lysed $48 \mathrm{~h}$ after transfection, and the levels of BECN1, LC3I and LC3II were detected by Western blot. (B) Cell viability assay. SH-SY5Y cells and SKNSH cells were transfected with expression vectors containing different genotypes of pri-miR-124. Relative cell viabilities were detected by MTT assay at 4 different time points. (C) Cell apoptosis assay. Cell apoptosis was detected using annexin V-FITC and propidium iodide (PI) staining and analyzed by flow cytometry. ${ }^{*} P<0.05,{ }^{* *} P<0.01$ vs miR-124-CC vector group.

ment syndromes caused by brain lesions during development. Cerebral palsy is caused by abnormal development or damage to the parts of the brain that control movement, balance, and posture. The risk factors for $\mathrm{CP}$ are mainly related to the external element, such as intrauterine infection, intrauterine growth restriction and perinatal stroke. However, genetic factors have also recently been confirmed as contributing to the susceptibility to cerebral damage and being related to the pathogenesis of CP. Recently, a genetic association study confirmed that nucleotide variations in protein coding genes are related to $\mathrm{CP}$, such as in IL-6 and CCL18 TNF-a ${ }^{[26-28]}$. In this study, we screened a more than 400-bp segment of the pri-miR-124-1, $-2,-3$ coding regions, and established this kind of association by confirming significant differences in the genotype distribution of rs3802169 and rs191727850 in $233 \mathrm{CP}$ patients and 256 healthy controls. The rare alleles T of rs3802169 and G of rs191727850 were found to be associated with an increased risk of $\mathrm{CP}(\mathrm{OR}=3.71,95 \% \mathrm{CI} 1.74-7.92$; OR=2.18, 95\% CI 1.36-3.49). Further analysis showed that the levels of mature miR-124 were down-regulated by the C-to-T change in vitro, which was consistent with the in vivo detection that the level of mature miR-124 was lower in $60 \mathrm{CP}$ patients with the CT genotype. We predicted the effect of the SNP rs3802169 on the secondary structure of miR-124. The $\mathrm{T}$ allele causes a change in the predicted secondary structure and a lower $\Delta \mathrm{G}$ compared with those with the $C$ allele. Therefore, we speculated that the SNP rs3802169 might influence the stability of the pri$\mathrm{miR}$, the efficiency of changing from pri-miR into pre-miR, or the efficiency of processing pre-miR into the mature miR. More importantly, the reduction of miR-124, as a consequence of the $\mathrm{C}$ to $\mathrm{T}$ change, further led to reduced efficient inhibition of the target genes, ITGB1, LAMC1 and BECN1, which may 
A

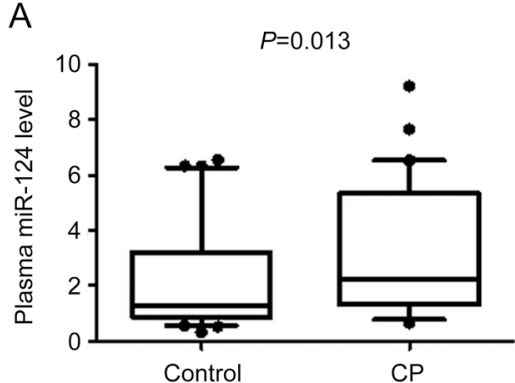

B

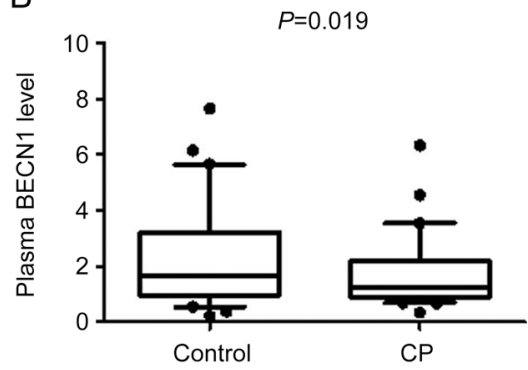

C

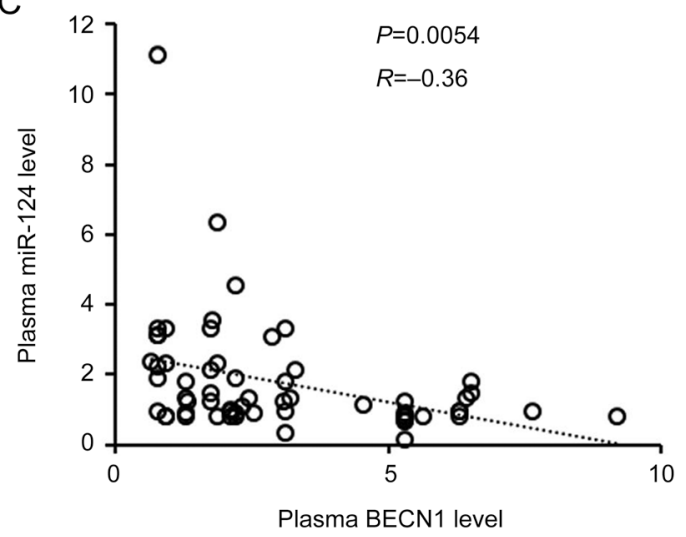

A

Nucleus
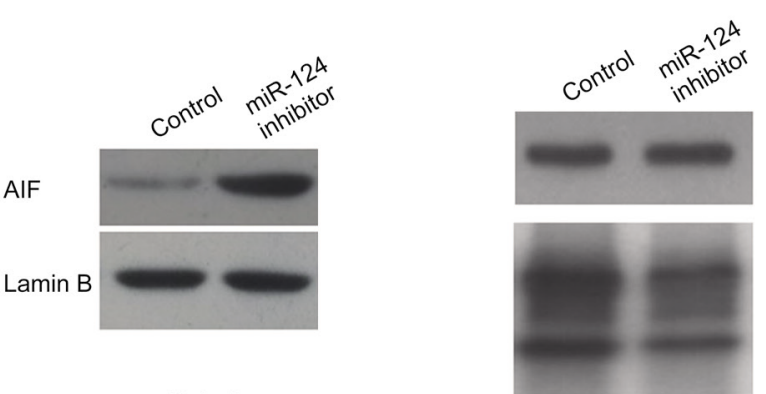

Cytoplasm

AIF

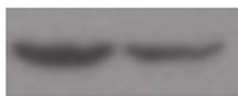

$\beta$-Actin

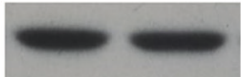

PARP1

PADPR

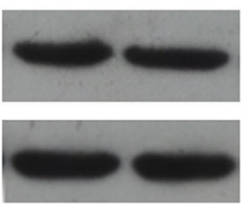

AIF

$\beta$-Actin
B

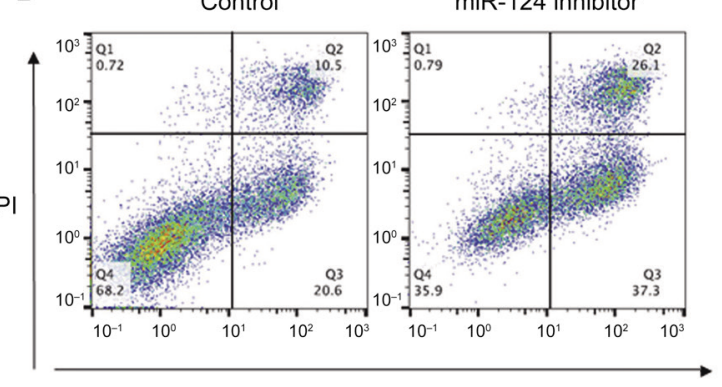

FITC-Annexin V

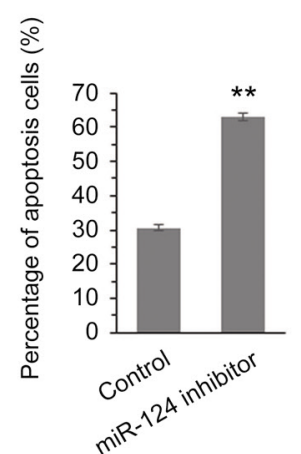

Figure 5. The plasma BECN1 level was negatively correlated with the plasma miR-124 level. (A) The plasma miR-124 levels in $60 \mathrm{CP}$ patients and 60 healthy controls were determined by qRT-PCR. (B) The plasma BECN1 levels of $60 \mathrm{CP}$ patients and 60 healthy controls were determined by ELISA. (C) The correlation between BECN1 level and miR-124 expression was analyzed using $\mathrm{X}^{2}$-analysis.
Figure 6. miR-124 reduction was related to AIF nuclear translocation. (A) The nuclear and cytoplasmic AIF contents of the control and miR124 inhibitor groups and the total contents of PARP1, PADPR and AIF protein were detected by Western blot. (B) Cell apoptosis percentage of SH-SY5Y cells was determined by flow cytometry. In addition, the graph shows the apoptosis percentages of SH-SY5Y cells, both the control and with the miR-124 inhibitor. ${ }^{* *} P<0.01$ vs control group. 
play important roles during the development of the nervous system. Thus, our data collectively suggested that one functional polymorphism in pri-miR-124-1 might contribute to the genetic predisposition to $\mathrm{CP}$ by disrupting the production of miR-124, which consequently interfered in the expression and function of target genes of miR-124.

In this study, we found that enhanced AIF nucleus translocation is related to the miR-124 level reduction under oxidative stress and is related to up-regulated cell apoptosis, which may provide another piece of evidence to explain the relationship between miR-124 and $\mathrm{CP}$.

In conclusion, we established the first association between SNPs in miR-124-1 and the predisposition to CP in one Chinese Han population by identifying one functional SNP in pri-miR-124-1. These findings may give insight into the understanding of RPL development and may create an opportunity to expand the approach to diagnosing and treating RPL.

\section{Abbreviation}

$\mathrm{AI}$, apoptosis inducing factor; $\mathrm{CP}$, cerebral palsy; ELISA, enzyme-linked immunosorbent assay; HWE, HardyWeinberg equilibrium; OR, odds ratio; SNPs, single nucleotide polymorphisms.

\section{References}

1 Wu YW, Croen LA, Vanderwerf A, Gelfand AA, Torres AR. Candidate genes and risk for CP: a population-based study. Pediatr Res 2011; 70: 642-6.

2 Wu YW, Escobar GJ, Grether JK, Croen LA, Greene JD, Newman TB. Chorioamnionitis and cerebral palsy in term and near-term infants. JAMA 2003; 290: 2677-84.

3 Nelson KB, Lynch JK. Stroke in newborn infants. Lancet Neurol 2004; 3: 150-8.

4 Gibson CS, Maclennan AH, Dekker GA, Goldwater PN, Sullivan TR, Munroe DJ, et al. Candidate genes and cerebral palsy: a populationbased study. Pediatrics 2008; 122: 1079-85.

5 Gibson CS, MacLennan AH, Goldwater PN, Haan EA, Priest K, Dekker $\mathrm{GA}$, et al. The association between inherited cytokine polymorphisms and cerebral palsy. Am J Obstet Gynecol 2006; 194: 674.e1-11.

6 Moreno-De-Luca A, Ledbetter DH, Martin CL. Genetic insights into the causes and classification of cerebral palsies. Lancet Neurol 2012; 11: 283-92.

7 Bi D, Chen M, Zhang X, Wang H, Xia L, Shang Q, et al. The association between sex-related interleukin- 6 gene polymorphisms and the risk for cerebral palsy. J Neuroinflammation 2014; 11: 100.

8 Krichevsky AM, King KS, Donahue CP, Khrapko K, Kosik KS. A microRNA array reveals extensive regulation of microRNAs during brain development. RNA 2003; 9: 1274-81.

9 Krichevsky AM, Sonntag KC, Isacson O, Kosik KS. Specific microRNAs modulate embryonic stem cell-derived neurogenesis. Stem Cells 2006; 24: 857-64.

10 Olde Loohuis NF, Nadif Kasri N, Glennon JC, van Bokhoven H, Hébert SS, Kaplan BB, et al. The schizophrenia risk gene MIR137 acts as a hippocampal gene network node orchestrating the expression of genes relevant to nervous system development and function. Prog
Neuropsychopharmacol Biol Psychiatry 2017; 73: 109-18.

11 Wang S, Xu Y, Li M, Tu J, Lu Z. Dysregulation of miRNA isoform level at 5 ' end in Alzheimer's disease. Gene 2016; 584: 167-72.

12 Gu H, Li H, Zhang L, Luan H, Huang T, Wang L, et al. Diagnostic role of microRNA expression profile in the serum of pregnant women with fetuses with neural tube defects. J Neurochem 2012; 122: 641-9.

13 Sanuki R, Onishi A, Koike C, Muramatsu R, Watanabe S, Muranishi Y, et al. miR-124a is required for hippocampal axogenesis and retinal cone survival through Lhx2 suppression. Nat Neurosci 2011; 14: 1125-34.

14 Jenn-Yah Y, Kwan-Ho C, Monika D, Robert C, Thompson D, Turner L. MicroRNA miR-124 regulates neurite outgrowth during neuronal differentiation. Exp Cell Res 2008; 314: 2618-33.

15 Liang XH, Kleeman LK, Jiang HH, Gordon G, Goldman JE, Berry G, et al. Protection against fatal Sindbis virus encephalitis by beclin, a novel Bcl-2-interacting protein. J Virol 1998; 72: 8586-96.

16 Allen Institute for Brain Science. 2004. Allen Brain Atlas. http:// www.brain-map.org.

17 Cao Y, Klionsky DJ. Physiological functions of Atg6/Beclin 1: a unique autophagy-related protein. Cell Res 2007; 17: 839-49.

18 Zhang F, Wang B, Long H, Yu J, Li F, Hou H, et al. Decreased miR-124$3 p$ expression prompted breast cancer cell progression mainly by targeting Beclin-1. Clin Lab 2016; 62: 1139-45.

19 Schwander M, Shirasaki R, Pfaff SL, Müller U. Beta1 integrins in muscle, but not in motor neurons, are required for skeletal muscle innervation. J Neurosci 2004; 24: 8181-91.

20 Radner S, Banos C, Bachay G, Li YN, Hunter DD, Brunken WJ, et al. $\beta 2$ and $\mathrm{\gamma} 3$ laminins are critical cortical basement membrane components: ablation of Lamb2 and Lamc3 genes disrupts cortical lamination and produces dysplasia. Dev Neurobiol 2013; 73: 20929.

21 Jazdzewski K, Murray EL, Franssila K, Jarzab B, Schoenberg DR, de la Chapelle A. Common SNP in pre-miR-146a decreases mature miR expression and predisposes to papillary thyroid carcinoma. Proc Natl Acad Sci U S A 2008; 105: 7269-74.

22 Saunders MA, Liang $\mathrm{H}$, Li WH. Human polymorphism at microRNAs and microRNA target sites. Proc Natl Acad Sci U S A 2007; 104: 3300-5.

23 Hu Y, Liu CM, Qi L, He TZ, Shi-Guo L, Hao CJ, et al. Two common SNPs in pri-miR-125a alter the mature miRNA expression and associate with recurrent pregnancy loss in a Han-Chinese population. RNA Biol 2011; 8: 861-72.

24 Sellier E, Surman G, Himmelmann K, Andersen G, Colver A, KrägelohMann I, et al. Trends in prevalence of cerebral palsy in children born with a birthweight of $2500 \mathrm{~g}$ or over in Europe from 1980 to 1998. Eur J Epidemiol 2010; 25: 635-42.

25 Aycicek A, Iscan A. Oxidative and antioxidative capacity in children with cerebral palsy. Brain Res Bull 2006; 69: 666-8.

26 Kallankari H, Huusko JM, Kaukola T, Ojaniemi M, Mahlman M, Marttila $\mathrm{R}$, et al. Cerebral palsy and polymorphism of the chemokine CCL18 in very preterm children. Neonatology 2015; 108: 124-9.

27 Hou R, Ren X, Wang J, Guan X. TNF-alpha and MTHFR polymorphisms associated with cerebral palsy in chinese infants. Mol Neurobiol 2015; 53: 6653-8.

28 Khankhanian P, Baranzini SE, Johnson BA, Madireddy L, Nickles D, Croen LA, et al. Sequencing of the IL6 gene in a case-control study of cerebral palsy in children. BMC Med Genet 2013; 14: 126. 